\title{
Article \\ Effect of Enhanced UV-B Radiation on fruit maturity and qual- ity, and leaf Photosynthesis in ' Guifei ' Mango
}

\author{
Xiaokai Lin ${ }^{1,2}$,Haizhi Liao ${ }^{1,2}$, Jingjia Du ${ }^{1,2}$, Junjie Peng ${ }^{1,2}$ and Kaibing Zhou ${ }^{1,2, *}$ \\ 1 College of Horticulture, Hainan University, Haikou 570228, China; linxiaokai@hainanu.edu.cn \\ 2 Engineering Research Center of Selecting and Breeding New Tropical Crops Varieties, Ministry of Educa- \\ tion, Haikou 570228, China \\ * Correspondence: zkb@hainanu.edu.cn (K.Z.)
}

\begin{abstract}
Background: Investigating the characteristics of photosynthetic physiological changes of leaves in Mangifera indica L. cv. 'GuIfei' under enhanced UV-B radiation, natural light exposed trees were regarded as control, and $96 \mathrm{~kJ} \cdot \mathrm{m}^{-2} \cdot \mathrm{d}^{-1}$ enhanced UV-B radiation was artificially simulated in the field; (2) Methods: The changes of fruit maturity and fruit quality, leaf net photosynthetic rate (Pn), photosynthetic pigments contents, photochemical reaction, activities of photosynthetic enzymes and their genes expressions were determined; (3) Results: Compared with CK, the percentage of mature fruits of the treatment was significantly increased, and fruit quality was better. The net photosynthetic rate $\left(\mathrm{P}_{\mathrm{n}}\right)$, the contents of photosynthetic pigment, Hill reaction activity and photochemical quenching coefficient $(\mathrm{qP})$ of the treatment leaves showed a significantly higher trend than CK. The activities of Rubisco and RCA, and the expression of Rubisco genes $r b c L$ and $r b c S$ were significantly increased; (4) Conclusions: $96 \mathrm{~kJ} \cdot \mathrm{m}^{-2} \cdot \mathrm{d}^{-1}$ enhanced UV-B radiation treatment improved Rubisco activity through increasing the expression of Rubisco genes $r b c L$ and $r b c S$, thereby enhancing the $\mathrm{CO}_{2}$-fixing capacity and dark reaction capacity of leaves. Based on this, it raised the net photosynthetic rate of leaves, which promoted the early maturity of 'Guifei' mango by the fast accumulating photosynthetic products.
\end{abstract}

Keywords: UV-B radiation; Mango; Fruit maturity; Fruit quality,Photosynthesis; Photosynthetic enzymes; Gene expression

\section{Introduction}

The ultraviolet radiation B region (UV-B,280-315 nm) is critical for plant growth and development, morphogenesis, adaptive orientation, photosynthesis, and secondary metabolism[1,2,3] . In recent years, the atmospheric ozone layer thinning leads to enhanced UV-B radiation reaching the biosphere[4]. At this time, UV-B radiation is called 'enhanced UV-B radiation'. Observations on a global scale show that the process of global ozone layer thinning is still intensifying[5], and thus Earth's organisms will have to deal with the enhanced UV-B radiation stress in the coming decades.

Currently, studies on the effects of enhanced UV-B radiation and its causes have been conducted in China and abroad mainly on food crops[6,7,8,9], algae[10], etc. One of the effects of enhanced UV-B radiation on plants are in photosynthesis, and in general, high dose of UV-B radiation leads to the decrease of chloroplast content, the destruction of photosynthetic system reaction center, the reductions of Hill reaction activity, photosynthetic system II (PSII) light use efficiency and net photosynthetic rate[11,12], It also greatly reduces the yield and quality of the cotton[13].

Some studies have also shown that enhanced UV-B radiation can have beneficial effects on plants, with appropriate enhanced UV-B radiation increasing the photosynthetic pigments contents of roasted tobacco[14], improving Rubisco enzyme activity[15], promoting the photosynthetic rate of Trichosanthis Fructus[16] and intensifing the UV-B radiation tolerance of grape by Increasing Antioxidant Enzyme Activities[17]

Mango (Mangifera indica L.) is an evergreen tree belonging to the family of Anacardiaceae, which is known as the ' king of tropical fruits ' in China. Hainan is one of the most important producing areas. Although it is located in the tropic area with low latitude and 
strong ultraviolet radiation, mango can well adapt to this environment.For the possibility of stronger UV-B radiation in the future, it is necessary to study the effect of the enhanced UV-B radiation on fruit development and leaf photosynthesis in mango. Recent studies showed that the enhanced UV-B radiation decresed yield, fruit quality, and photosynthetic rate in ' Tainong 1 ' mango and ' Jinhuang ' mango[18,19,20]. At present, there is no report on the effects of enhanced UV-B radiation on the fruit development and leaf photosynthesis in ' Guifei ' mango, another major cultivar in Hainan province.

In this paper, ' Guifei ' mango was used as the experiment materials, and the enhanced UV-B radiation treatment of $96 \mathrm{~kJ} \cdot \mathrm{m}-2 \cdot \mathrm{d}-1$ was simulated in the field. Mature fruit ratio, fruit quality indexes including content of soluble sugar, titrable acid, total soluble solid, and vitamin C, and leaf photosynthetic parameters were measured to study the effect of enhanced UV-B radiation on the fruit maturity and quality, and leaf photosynthetic capacity in 'Guifei' mango. Our results reveals the reponse of 'Guifei' mango to enhanced UV-B radiation, which is helpful for improving the tolerance to UV-B stress in mango in the future.

\section{Materials and methods}

\subsection{Experimental materials}

The trial point was located in a collectively owned mango orchard of Liaoci Village, Yingzhou Town, Lingshui Li Autonomous County, Hainan Province, with tropical and monsoonal climate. The annual precipitation is $1500-2500 \mathrm{~mm}$, and the annual average temperature is $25.2{ }^{\circ} \mathrm{C}$. We selected 1016 -year-old trees of 'Guifei' mango, which were robust and uniform in growth and free from diseases and pests, with a spacing of $3 \mathrm{~m} \times 5 \mathrm{~m}$. The technique of yield regulation is implemented with Qingming Festival as the expected harvesting period, with the flowering period in late December, the flowering period in early January, the first physiological fruiting period in mid-January, the second physiological fruiting period in late January, the fruiting period in early February, the fruit expansion period in early to mid-April, and the ripening period in late April to early May.

\subsection{Experimental design}

Natural light (average daily radiation dose of about $83.47 \mathrm{~kJ} \cdot \mathrm{m}^{-2} \cdot \mathrm{d}^{-1}$ ) was used as control (CK), and $96 \mathrm{~kJ} \cdot \mathrm{m}-2 \cdot \mathrm{d}-1$ artificially simulated enhanced UV-B radiation was set up in the field as treatment. The field experiment was designed with single tree plot and five replications. Treatment was carried out from full flowering (late December) to fruit ripening period (mid May). A trellis was set up in the test garden and with four UV lamps (40 $\mathrm{W}$ per lamp, radiation dose $24 \mathrm{~kJ} \cdot \mathrm{m}^{-2} \cdot \mathrm{d}^{-1}$ ) crossing at the top of the test trees maintaining a $30 \mathrm{~cm}$ median position, and the lamps were turned on daily from 8:00 to 17:00 (treatment was stopped on cloudy and rainy days) for 5 months of continuous treatment.

\subsection{Sampling and sample handling}

The first sample was taken on December 27, 2019, and five mature leaves were randomly picked near the top of the canopy and sampled for photosynthetic parameters measurement. The second sampling were carried out on January 17,2020, and yet the sampling was forced to stop for a period of time due to the new coronavirus pneumonia epidemic. The photosynthetic parameters were determined and sampled again until April 22,29 and May 7,15 and 22, respectively. Among them, the yield per tree was investigated in the field on April 22, 29 and May 7(134d, 141d, and 150d after flowering), and the mature fruit ratio was calculated. Ten fully mature fruits (treatment) and the highest mature fruits (CK) were randomly picked at the periphery of the middle crown as test samples. When the fruits of CK matured to full maturation in the laboratory, the fruit samples of treatment and CK were prepared to measure the fruit qualities indexes. The harvested leaf samples and fully ripe fruit samples were promptly snapped frozen in liquid nitrogen tanks and stored at $-80{ }^{\circ} \mathrm{C}$ in an ultra-low temperature refrigerator.

\subsection{Determination indicators and methods}




\subsubsection{Determination of ripe mango fruit ratio}

The yield per tree was investigated in the field on $134 \mathrm{~d}, 141 \mathrm{~d}$, and $150 \mathrm{~d}$ after flowering and the fruit maturation rate was investigated according to the standard of complete fruit maturation with yellow to red skin.

\subsubsection{Determination of Mango Quality Index}

The soluble sugar content and $\mathrm{Vc}_{\mathrm{c}}$ content of pulp were measured according to the method described by Li[21]. The soluble solids and titratable acid content of pulp were measured by a sugar acid meter (ATAGO, PAL-BXIACID15, Tokyo, Japan). The sugar acid ratio of fruit was the ratio of soluble sugar content to titratable acid content, and the solid acid ratio of fruit was the ratio of soluble solids content to titratable acid content.

\subsubsection{Measurement of leaf net photosynthetic rate}

The net photosynthetic rate $\left(\mathrm{P}_{\mathrm{n}}\right)$ of leaves was measured by Photosynthesis Analyzer (YAXIN LIYI, Yaxin-1101, Beijing, China)

2.4.4. Determination of chlorophyll fluorescence quenching coefficient and physiological and biochemical indicators in leaves

Leaf photosynthetic pigments contents were determined by Arnon method[22], and chlorophyll $\mathrm{a}+\mathrm{b}$ content and $\mathrm{a} / \mathrm{b}$ values were calculated. Determination of chlorophyll fluorescence quenching coefficients using a dual-channel modulated chlorophyll fluorometer (Zealquest Scientific Technology, DUAL-PAM-100, Shanghai, China). The Hill reaction activity was determined by literature method[23].

2.4.5. Determination of carbon assimilation related enzymes activities and genes expressions in leaves

Enzyme Activities of Rubisco Activase (RCA) and Ribulose-1,5-diphosphate Carboxylase/Oxygenase (Rubisco) by ELISA Kit (Sangon Biotech, Shanghai, China). Total RNA was extracted by UNIQ-10 column Trizol total RNA extraction kit (Sangon Biotech, B511321, Shanghai, China). Maxima Reverse Transcriptase (Vazyme, EP0307, Nanjing, China) was used for reverse transcription, and 2X SG Fast qPCR Master Mix (Vazyme, B639273, Nanjing, China) was used for real-time quantitative PCR (Q-PCR). The relative quantitative calculation was performed by $2^{-\Delta \Delta C t}$ method. Primers for amplifying $r b c L$ and $r b c S$ were designed using Primer3 ( https://primer3.ut.ee/) online tool, and the reference gene was Actin gene[24]. The primers designed in this study are listed as follows, $r b c L(\mathrm{~F}$ ACGCCGGTACAGTAGTAGGT, R GAATCCAGTCCAACCACG), rbcS(F CCTCTCCTACCTCCCTCCTC, R GCACATGTCCCACCTCATCA ).

\subsection{Data statistics}

SAS software was used for statistical analysis. The ANOVA process was used for variance analysis on the dynamic changes of each indicator, and LSD method was used for multiple comparative analysis $(\mathrm{P}<0.05)$. The TTEST process was used to test the significance of the difference between the treatment and the control in each indicator.

\section{Results and analysis}

\subsection{Effect of enhanced $U V-B$ radiation on mature fruit ratio}

As seen in Figure 1 and 2, the mature fruit ratio was significantly higher in all treatments than in CK during the period from . The mature fruit ratio of CK was almost 0 , and 6.77\% higher in the treatment than in CK on $134 \mathrm{~d}$ after flowering, The treatment was $37.83 \%$ higher than CK on $141 \mathrm{~d}$ after flowering, which showed the maximum difference. On $150 \mathrm{~d}$ after flowering, the treatment was $17.52 \%$ higher than CK. It can be seen that the enhanced UV-B radiation treatment of $96 \mathrm{~kJ} \cdot \mathrm{m}^{-2 \cdot} \mathrm{d}^{-1}$ promoted fruit ripening of 'Guifei' mango. 

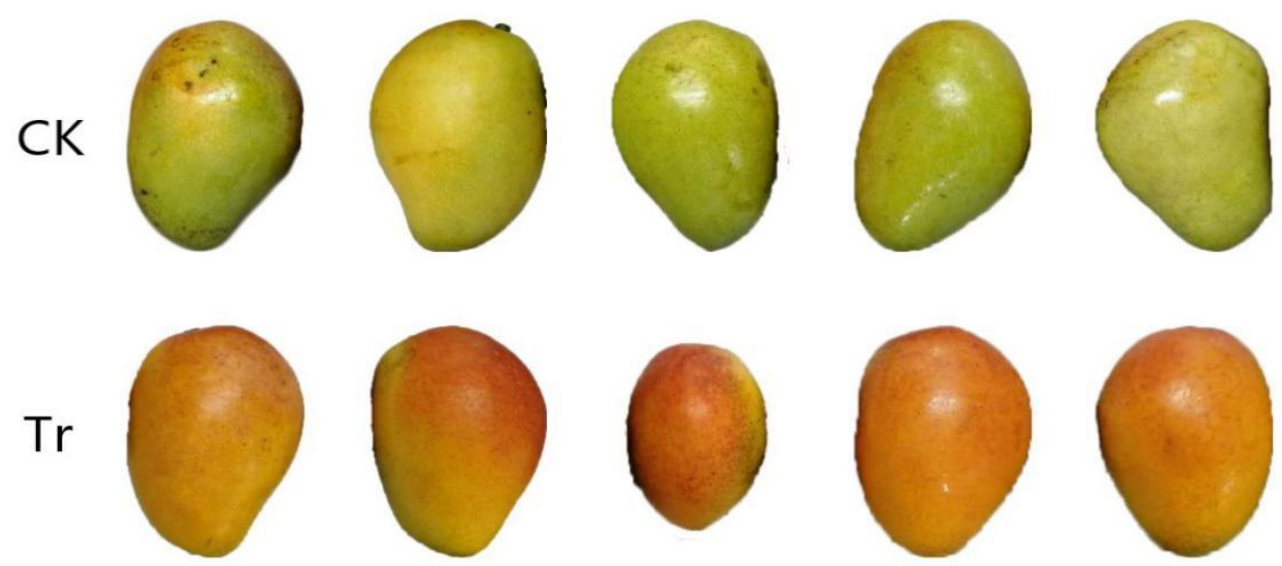

Figure 1. First batch of mangoes harvested on $134 \mathrm{~d}$ after flowering.

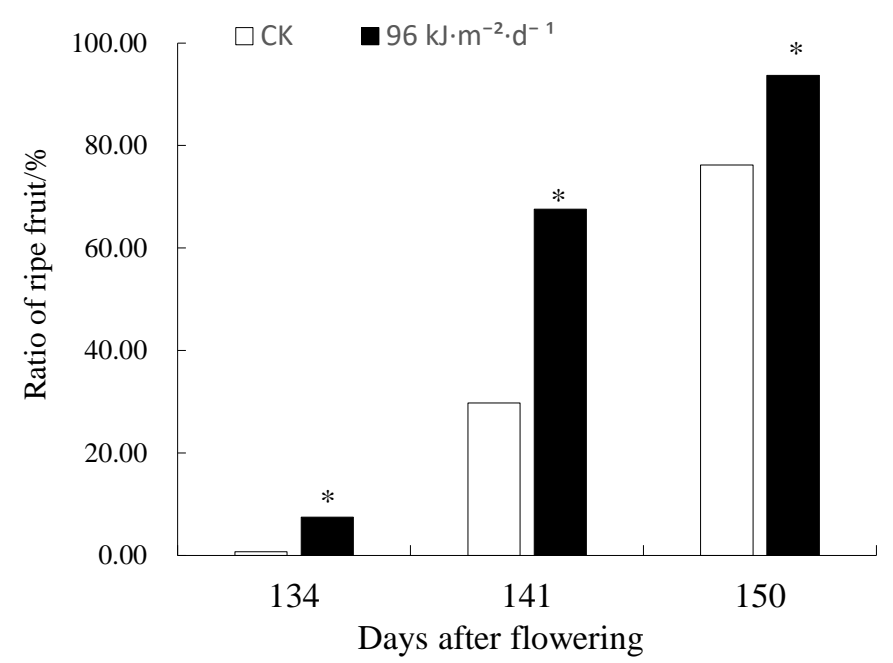

Figure 2. The effects of enhanced UV-B radiation on mature fruit ratio.(Note: ${ }^{*}$ in the figure indicates significant difference between treatment and control $(\mathrm{P}<0.05)$. The same below.).

\subsection{Effects of Enhanced UV-B Radiation on Fruit Quality}

Table 1 and table 2 showed that the soluble sugar, soluble solids content, sugar-acid ratio and solid-acid ratio of the flesh treated on $134 \mathrm{~d}$ after flowering were significantly higher than those of $\mathrm{CK}$, and titratable acid content of the treatment was significantly lower than that of $\mathrm{CK}$, and there was no significant difference in other time. There was no significant difference in vitamin C content between the treatment and CK on $134 \mathrm{~d}$ and $141 \mathrm{~d}$ after flowering, and the content of $\mathrm{Vc}$ in the treatment flesh was significantly higher than that in CK on on $150 \mathrm{~d}$ after flowering. Therefore, there was no significant difference between the treatment and CK in fruit qualities during the period on $141 \mathrm{~d}$ and $150 \mathrm{~d}$ after flowering. The fruit quality of the treatment on $134 \mathrm{~d}$ after flowering was significantly better than that of $\mathrm{CK}$, which might be due to its early maturity.

Table 1. Comparison of soluble sugar, titratable acid, soluble solids content comparison between treatment and control.

\begin{tabular}{|c|c|c|c|c|c|c|c|c|c|}
\hline & \multicolumn{3}{|c|}{ Soluble sugar content $/ \%$} & \multicolumn{3}{|c|}{ Titratable acid content $/ \%$} & \multicolumn{3}{|c|}{ Soluble solid conrent $/ \%$} \\
\hline & $134 d$ & $141 d$ & $150 \mathrm{~d}$ & $134 d$ & $141 d$ & $150 \mathrm{~d}$ & $134 d$ & $141 d$ & $150 \mathrm{~d}$ \\
\hline CK & $9.07 \pm 0.60$ & $23 \pm 0$ & $6 \pm 0$ & $2 \pm 0.35$ & $5 \pm 0$ & $.35 \pm 0.2$ & $41 \pm 1$ & $3.95 \pm 1$ & $.43 \pm 1$ \\
\hline
\end{tabular}

Note: The data in the table are "mean \pm standard deviation", and there are significant differences between the data in the table and the treatment with ${ }^{*}$ and the control $(\mathrm{P}<0.05)$. The same below. 
Table 2. Comparison of Sugar - acid ratio, solid - acid ratio, vitamin C content between treatment and control.

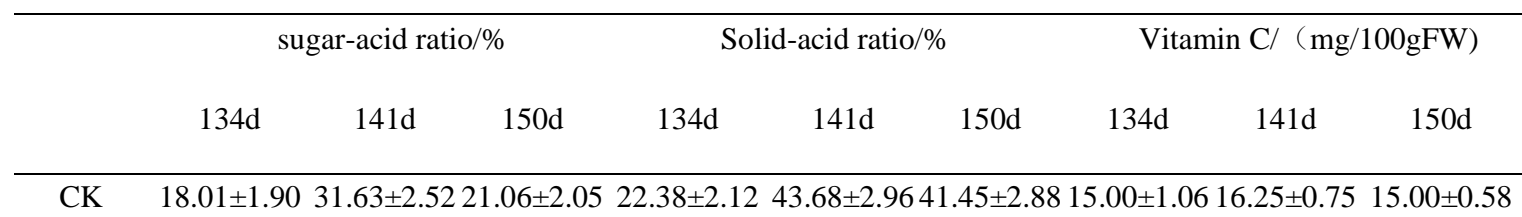

\subsection{Effects of enhanced $U V-B$ radiation on leaf photosynthetic rate}

As seen in Figure 3, the Pn of both treatment and CK showed a similar "M" type dynamic trend, with no significant change from December 27 to January 17, a significant decrease until April 22, and then a significant increase until May 7, followed by a significant decrease until May 22. At the same time, compared with CK, the $P_{n}$ of the treatment was significantly higher on April 22, April 29 and May 7 than that of CK. Visible, $96 \mathrm{~kJ} \cdot \mathrm{m}^{-}$ $2 \cdot \mathrm{d}^{-1}$ enhanced UV-B radiation treatment showed a trend to promote photosynthesis in the leaves of 'GuIfei' mango.

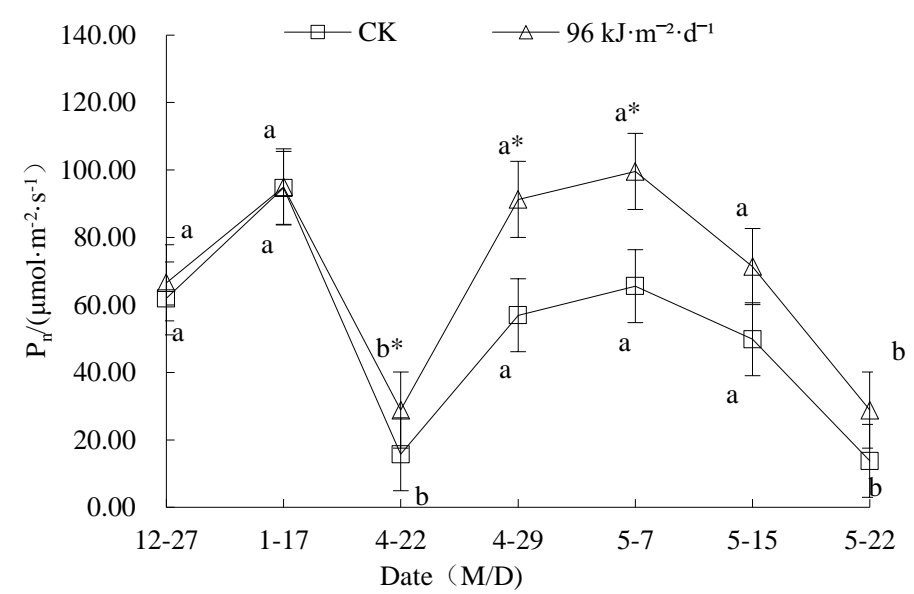

Figure 3. The effects of the enhanced UV-B radiation on the net photosynthetic rate $\left(P_{n}\right)$ in leaves.(Note: The Chinese and English letters of the line chart indicate the significant difference of the dynamic changes of different dates, different English letters indicate significant difference, the same letter means not significant. $(\mathrm{P}<0.05)$. The same below.).

\subsection{Effects of enhanced UV-B radiation on photosynthetic pigment content in leaves}

The effects of enhanced UV-B radiation treatment on photosynthetic pigment content in leaves as shown in table 3, there were significant differences between treatment and CK in the dynamic change trend and the same time.

The contents of chlorophyll $\mathrm{a}, \mathrm{b}$ and $\mathrm{a}+\mathrm{b}$ in CK had no significant change before May 15 , and then increased significantly. The contents of chlorophyll $a, b$ and $a+b$ in CK had no significant change from December 27 to April 22, and increased significantly after April 22. Chlorophyll $\mathrm{a} / \mathrm{b}$ of $\mathrm{CK}$ had no significant change before January 17 , and then showed a downward trend; the treatment showed no significant change until May 7 and a decreasing trend afterwards. The carotenoid content of CK did not change significantly from December 27 to May 15, but increased significantly after May 15. The treatment had no significant change before April 22 and then increased significantly. The chlorophyll a content of the treatment was significantly higher than that of the control on May 7 and 15, with no significant differences at the rest of the day. There was no significant difference in chlorophyll $b$ content between the treatment and the control before April 22, and then it was significantly higher than that of the control. The chlorophyll $a+b$ and $a / b$ of the treatment were significantly higher than those of the control on April 22 and May 15, and there was no significant difference in the rest time. The carotenoid content of the treatment 
was significantly higher than that of the control on April 22 and May 15, and there was no significant difference in the other time.

Table 3. The effects of enhanced UV-B radiation on photosynthetic pigment contents in mango leaves.

\begin{tabular}{|c|c|c|c|c|c|c|c|}
\hline & & $12 / 27$ & $1 / 17$ & $4 / 22$ & $5 / 07$ & $5 / 15$ & $5 / 22$ \\
\hline \multirow{2}{*}{ Chlorophylla/(mg $\left.\cdot \mathrm{g}^{-1}\right)$} & $\mathrm{CK}$ & $1.55 \pm 0.056 \mathrm{a}$ & $1.61 \pm 0.074 \mathrm{a}$ & $1.48 \pm 0.039 a$ & $1.63 \pm 0.060 \mathrm{a}$ & $1.67 \pm 0.060 \mathrm{a}$ & $1.97 \pm 0.075 b$ \\
\hline & $\operatorname{Tr}$ & $1.59 \pm 0.049 \mathrm{~cd}$ & $1.61 \pm 0.100 \mathrm{~cd}$ & $1.63 \pm 0.082 \mathrm{~d}$ & $1.83 \pm 0.034 b c^{*}$ & $1.87 \pm 0.048 b c^{*}$ & $2.12 \pm 0.057 \mathrm{a}$ \\
\hline \multirow{2}{*}{ Chlorophyllb/(mg. g-1) } & CK & $0.42 \pm 0.022 b$ & $0.46 \pm 0.044 \mathrm{ab}$ & $0.46 \pm 0.011 \mathrm{ab}$ & $0.51 \pm 0.016 \mathrm{ab}$ & $0.49 \pm 0.037 \mathrm{ab}$ & $0.55 \pm 0.024 \mathrm{a}$ \\
\hline & $\operatorname{Tr}$ & $0.44 \pm 0.041 \mathrm{~cd}$ & $0.48 \pm 0.036 \mathrm{~cd}$ & $0.46 \pm 0.031 \mathrm{~d}$ & $0.56 \pm 0.017 b c^{*}$ & $0.54 \pm 0.020 b^{*}$ & $0.60 \pm 0.023 \mathrm{a}^{*}$ \\
\hline \multirow{2}{*}{ Chlorophyll $\mathrm{a}+\mathrm{b}$} & CK & $1.90 \pm 0.073 \mathrm{a}$ & $2.06 \pm 0.110 \mathrm{a}$ & $1.97 \pm 0.071 \mathrm{a}$ & $2.20 \pm 0.075 \mathrm{a}$ & $2.16 \pm 0.082 \mathrm{a}$ & $2.52 \pm 0.098 b$ \\
\hline & $\operatorname{Tr}$ & $2.04 \pm 0.068 \mathrm{a}$ & $2.10 \pm 0.133 \mathrm{a}$ & $2.09 \pm 0.089 a$ & $2.39 \pm 0.051 b$ & $2.41 \pm 0.063 b^{*}$ & $2.71 \pm 0.070 \mathrm{~b}$ \\
\hline \multirow{2}{*}{ Chlorophyll a/b } & $\mathrm{CK}$ & $3.80 \pm 0.155 \mathrm{a}$ & $3.67 \pm 0.134 \mathrm{a}$ & $3.21 \pm 0.052 b$ & $3.26 \pm 0.063 b$ & $3.55 \pm 0.148 b$ & $3.65 \pm 0.095 b$ \\
\hline & $\operatorname{Tr}$ & $4.04 \pm 0.338 \mathrm{a}$ & $3.46 \pm 0.093 \mathrm{a}$ & $3.63 \pm 0.139 \mathrm{ab}^{*}$ & $3.31 \pm 0.065 b$ & $3.49 \pm 0.090 \mathrm{~b}$ & $3.63 \pm 0.175 \mathrm{ab}$ \\
\hline \multirow{2}{*}{ Carotenoid/mg. $\mathrm{g}^{-1}$} & CK & $0.35 \pm 0.014 \mathrm{c}$ & $0.39 \pm 0.014 b c$ & $0.36 \pm 0.011 \mathrm{c}$ & $0.40 \pm 0.015 b c$ & $0.44 \pm 0.020 \mathrm{~b}$ & $0.49 \pm 0.016 \mathrm{a}$ \\
\hline & $\operatorname{Tr}$ & $0.36 \pm 0.019 \mathrm{~d}$ & $0.37 \pm 0.020 \mathrm{~d}$ & $0.41 \pm 0.018 \mathrm{~d}^{*}$ & $0.43 \pm 0.008 b c$ & $0.49 \pm 0.012 \mathrm{ab} *$ & $0.52 \pm 0.015 \mathrm{a}$ \\
\hline
\end{tabular}

Note: The letters Tr. Symbolize the treatment $96 \mathrm{~kJ} \cdot \mathrm{m}^{-2} \cdot \mathrm{d}^{-1} \mathrm{UV}-\mathrm{B}$ radiation. The data in the table followed by $*$ indicates that there is a significant difference between the treatment and the control $(\mathrm{p}<0.05)$; The data followed by different English letters indicated significant differences, while those not followed by letters or the same letters indicated insignificant differences $(\mathrm{P}<0.05)$. The same below.

\subsection{Effect of enhanced UV-B radiation on Hill reaction of leaves}

As seen in Figure 4, the trends of Hill's response activity were different between the treatment and control, with the control showing essentially no significant change and the treatment showing an increasing and then decreasing trend.

The treatments were not significantly different from the control until January 7; Treatments were significantly higher than controls on 22 April, 7 and 15 May. Therefore, the treatment promoted the photolysis of water, which might further promote the photoreaction.

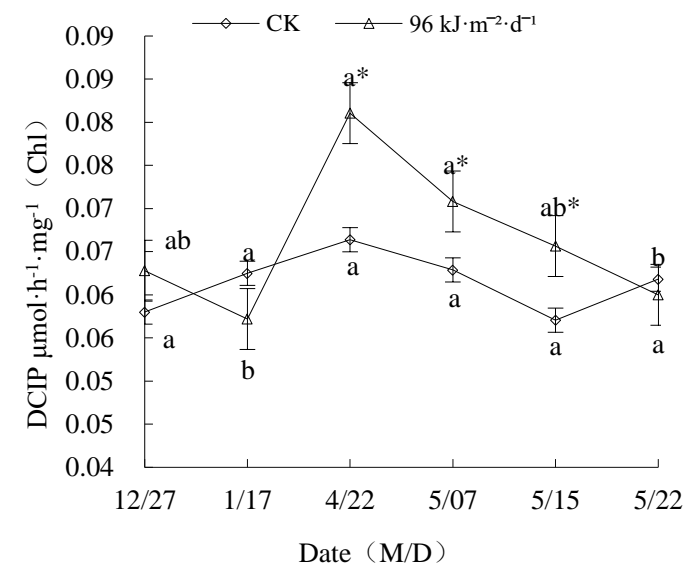

Figure 4. The effects of the enhanced UV-B radiation on the activity of Hill reaction in leaves. 


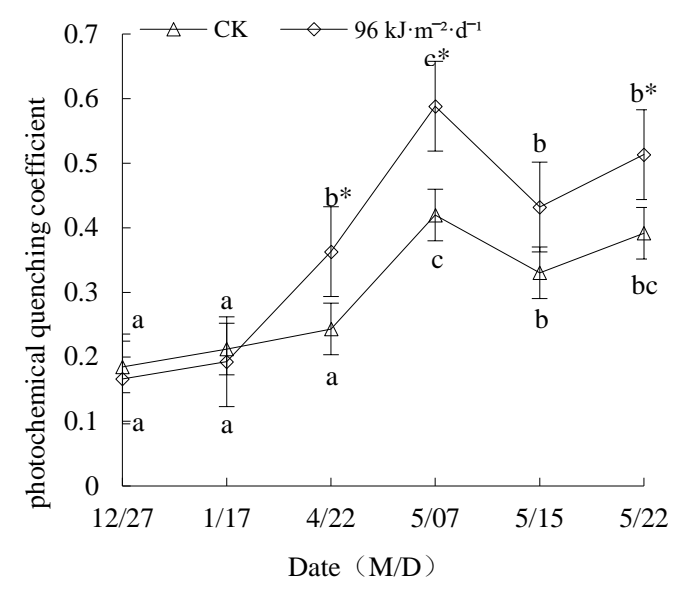

Figure 5. The effects of the enhanced UV-B radiation on photochemical quenching coefficient in leaves.

\subsection{Effect of enhanced $U V-B$ radiation on photochemical quenching coefficient $(q P)$ of leaves}

It can be seen from Fig.5 that the qP of both treatment and control showed a trend of increasing, then decreasing and then increasing. The treatments were significantly higher than the control on April 22, May 7 and 22, with no significant differences at the rest of the time. Thus, the treatment showed a trend of promoting electron transfer in the photosynthetic chain, which may further promote the light reaction.

\subsection{Effect of enhanced $U V-B$ radiation on leaf dark response}

\subsubsection{RCA and Rubisco activities}

As shown in Fig. 6, the activity of Rubisco activated enzyme (RCA) increased significantly from January 17 to April 22, and then tended to be flat. The control showed a gentle first and then decreased signfificantly on May 7, and then tended to be gentle. The RCA activity of the treatments was significantly higher than the control on April 22, May 7, 15 and 22 .

It can be seen from Fig. 7 that the activity of 1,5-diphosphate carboxylase (Rubisco) in the treatment and CK showed a similar trend of decreasing, then increasing, then reducing and then rising. On May 7, 15 and 22, the Rubisco activity of the treatments was significantly higher than that of the control. It can be seen that the enhanced UV-B radiation treatment showed a tendency to significantly increase leaf RCA activity and Rubisco activity, which promoted the assimilation of $\mathrm{CO}_{2}$.

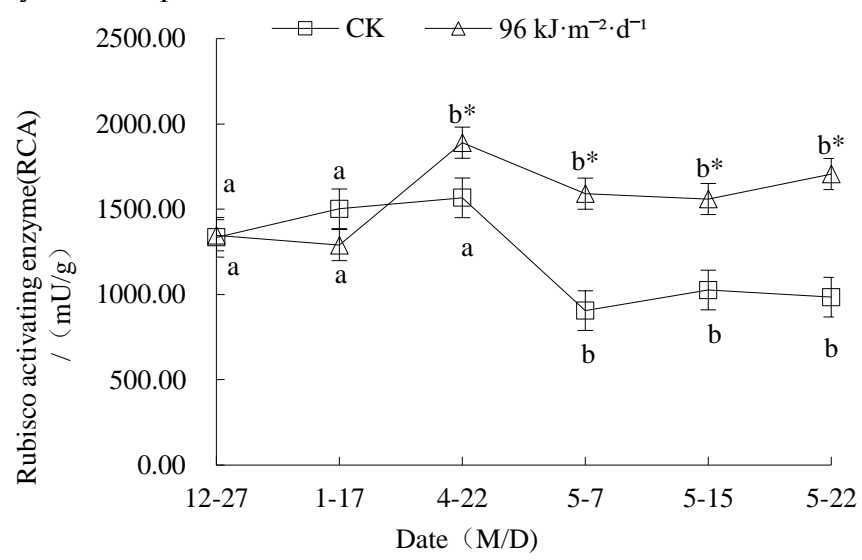

Figure 6. The effects of the enhanced UV-B radiation on RCA in leaves. 


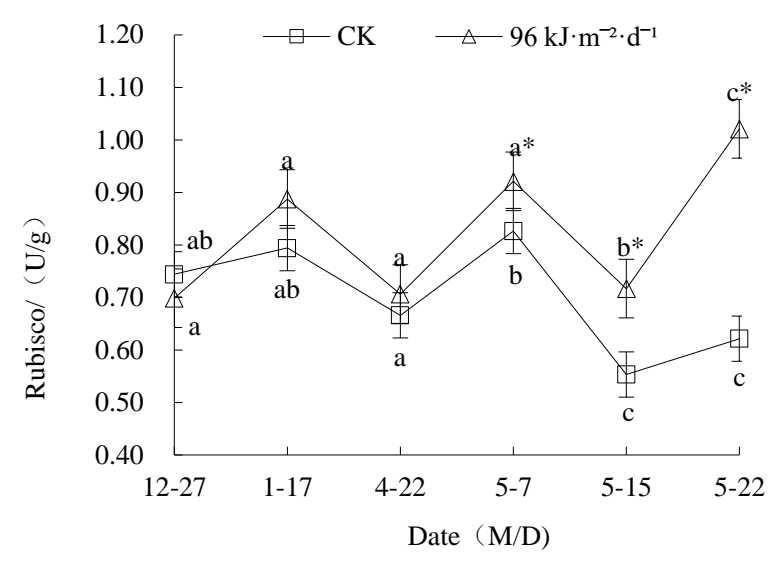

Figure 7. The effects of the enhanced UV-B radiation on Rubisco in leaves.

\subsubsection{Relative expression levels of rbcL and rbcS}

It can be seen from Fig. 8 that the relative expression level of the large subunit encoding gene $(r b c L)$ of the control 1,5 - diphosphate ribulose carboxylase (Rubisco) showed a flat trend, which increased significantly from April 22 to May 15, and then decreased significantly. The relative expression levels of the treatments first showed a gentle trend, after January 17 began to rise significantly to May 15, and then decreased significantly. The expression level of $r b c L$ in the treatment was significantly higher than that in the control on April 22, May 15 and 22.

It can be seen from Fig. 9 that the relative expression level of the small subunit encoding gene $(r b c S$ ) of the control Rubisco showed a flat trend and significantly increased after May 15; The relative expression level showed a gentle trend, and increased significantly after January 17 . At the same time, the expression level of $r b c S$ in the treatment was significantly higher than that in the control on April 22 and May 15.

It can be seen that $96 \mathrm{~kJ} \cdot \mathrm{m}^{-2} \cdot \mathrm{d}^{-1} \mathrm{UV}-\mathrm{B}$ radiation enhanced the expression of Rubisco large subunit ( $r b c L$ ) and small subunit ( $r b c S$ ) of Rubisco.

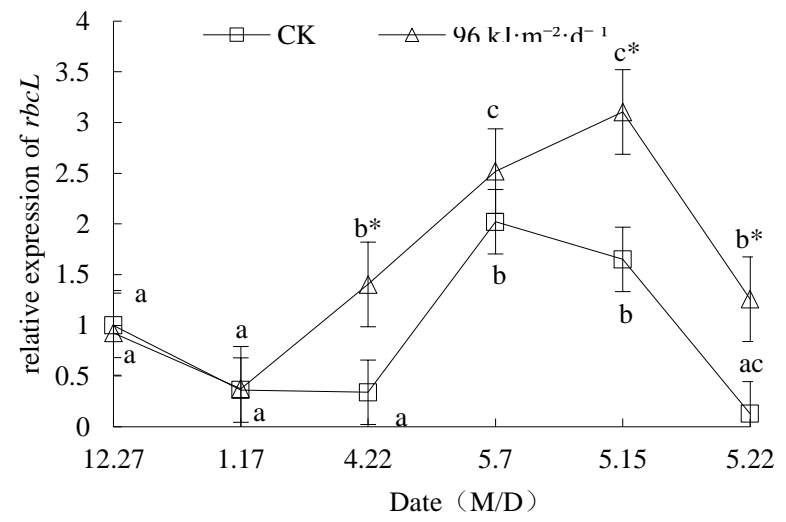

Figure 8. The effects of the enhanced UV-B radiation on the relative expression of Rubisco gene $(r b c L)$ in leaves.

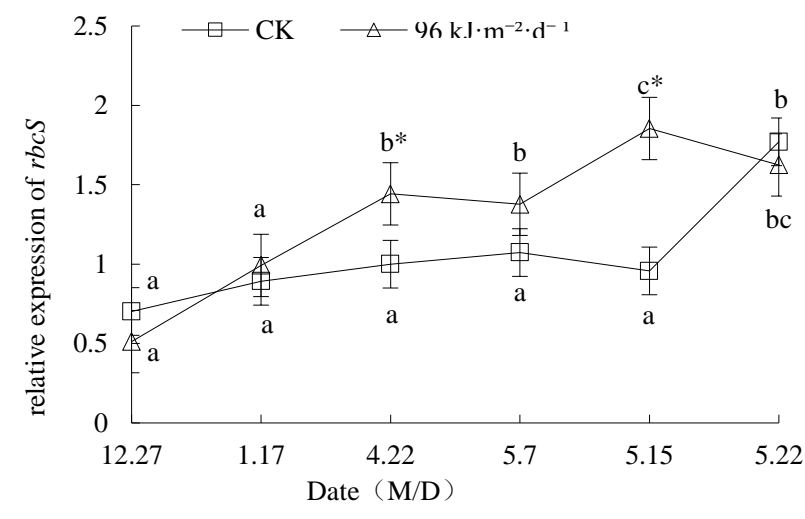


Figure 9. The effects of the enhanced UV-B radiation on the relative expression of Rubisco gene $(r b c S)$ in leaves.

\section{Discussion}

4.1. Effects of Enhanced UV-B Radiation on Photosynthetic Physiology of Leaves, mature fruit ratio and fruit qualiti.

As an indicator to measure the intensity of photosynthesis, photosynthetic pigment content can reflect the changes of organs and tissues and the intensity of photosynthetic physiological ability of plants under environmental stress[25]. Depending on the crop species, Photosynthetic pigment content increases or decreases with increasing UV-B radiation intensity depending on crop varieties[26]. Photosynthetic pigment content of ' Guifei ' mango under $96 \mathrm{~kJ} \cdot \mathrm{m}^{-2} \cdot \mathrm{d}^{-1}$ enhanced UV-B radiation changed significantly, and chlorophyll a, b, carotenoid content, chlorophyll $\mathrm{a}+\mathrm{b}$ and $\mathrm{a} / \mathrm{b}$ values increased significantly, chlorophyll $a+b$ and $a / b$ values were positively correlated with photosynthetic rate[27]. It may be that the intensity of UV-B radiation did not reach the inhibition threshold of ' Guifei ' mango, but it was beneficial to the enhancement of its photosynthetic capacity, which was consistent with the results of Yang[28]. And the chlorophyll a/b value was positively correlated with the degree of thylakoid stacking, which reflected that enhanced UV-B radiation treatment was beneficial to the optimization of chloroplast thylakoid structure. Therefore, it is speculated that enhanced UV-B radiation may accumulate more assimilation by optimizing the stacking structure of chloroplast thylakoids, increasing the content of photosynthetic pigments and optimizing the composition ratio. Carotenoids, a photoprotective pigment, dissipate absorbed UV-B radiation as well as scavenge free radicals[29], and elevated carotenoid content may be a protective mechanism initiated by the leaves after enhanced UV-B radiation treatment, while enhancing the ability of the leaves to capture light energy.

Photosynthesis is the material and energy basis of plant growth and development, which is very sensitive to environmental changes[30]. Numerous studies have shown that enhanced UV-B radiation adversely affects plants, leading to a decrease in photosynthetic rate [31,32]. The study found that $C 3, C 4$ and CAM plants showed different performance under enhanced UV-B radiation[33] , indicating that different species of plants had different enhanced responses to UV-B radiation. It was found that the photosynthetic rate of ' Guifei ' mango treated by enhanced UV-B radiation was higher than that of 'Guifei ' mango without UV-B radiation. Previous studies on ' Tainong NO. 1 ' mango and 'Jinhuang' mango have found that enhanced UV-B radiation inhibits photosynthesis, resulting in a decrease in photosynthetic rate[19,20] , but the results in this paper are opposite, which may be caused by the differences in UV-B radiation resistance among different mango cultivars.

Li found that UV-B radiation promotes the ripening of highbush blueberry[34] . Some studies also found that under UV-B radiation of $150 \mu \mathrm{W} / \mathrm{cm}^{2}$ the fruit quality of grape berry was significantly improved, which was consistent with the results of our study [35]. The results showed that $96 \mathrm{~kJ} \cdot \mathrm{m}^{-2} \cdot \mathrm{d}^{-1}$ enhanced UV-B radiation treatment could promote early ripening and improve fruit quality of ' Guifei ' mango. It is speculated that the reason may be that enhanced UV-B radiation can enhance the photosynthetic function of leaves, thereby promoting fruit growth and early ripening.

\subsection{Photoreaction changes}

When most plants are subjected to enhanced UV-B radiation stress, they will weaken the activity of PS II reaction center [36], damage the PS II electron transport system and inhibit the primary photosynthetic reaction [37,38], decreased Hill reaction activity and photochemical quenching coefficient $(\mathrm{qP})$, resulting in reduced formation of assimilation and decreased net photosynthetic rate. However, the results showed that $96 \mathrm{~kJ} \cdot \mathrm{m}^{-2} \cdot \mathrm{d}^{-1} \mathrm{en}-$ hanced UV-B radiation treatment of 'Guifei' mango leaves Hill reaction activity and qP showed an upward trend, contrary to previous studies. The Hill reaction activity was positively correlated with photosynthetic capacity[39]. Therefore, it is speculated that enhanced UV-B radiation may enhance the photoreaction activity of leaves, thus creating 
energy conditions for dark reaction to fix more $\mathrm{CO} 2$, and further enhancing the photosynthetic capacity.

\subsection{Dark reaction changes}

Rubisco (ribulose 1,5-bisphosphate carboxylase/oxygenase) is a key enzyme in photosynthetic carbon assimilation, which catalyzes both carbon reduction in photosynthesis and carbon oxidation in photorespiration[40]. Among them, Rubisco enzyme is composed of eight $56 \mathrm{kD}$ large subunit $(r b c L)$ and eight $14 \mathrm{kD}$ small subunit ( $r b c S$ ), which are encoded by chloroplast gene and nuclear gene. Although $r b c L$ contains the active site of Rubisco total enzyme, recent studies have shown that $r b c S$ has a very important effect on the regulation of Rubisco total enzyme activity and protein content[41].Abiotic stress leads to a decrease in Rubisco enzyme activity, large subunit and small subunit protein content, which in turn inhibits photosynthetic efficiency[42].It has been shown that enhanced UV$B$ radiation caused a decline in Rubisco large subunit gene expression and Rubisco enzyme carboxylation activity in' Tainong 1 ' mango[20], but little has been reported about small subunit and Rubisco activating enzymes in mango. Rubisco in the passive state must be activated by Rubisco activating enzyme (RCA) to catalyze its activity[43], that is the activity of Rubisco in plants depends on the activation degree of RCA. The activation of Rubisco is a key factor determining the carbon assimilation efficiency of leaves. In this paper, the Rubisco and RCA enzyme activities of leaves of 'Guifei' fruit trees treated with enhanced UV-B radiation at $96 \mathrm{~kJ} \cdot \mathrm{m}^{-2} \cdot \mathrm{d}^{-1}$ were significantly increased, which was beneficial to improve the $\mathrm{CO} 2$ assimilation efficiency and photosynthetic electron transfer efficiency, and the significantly increased RCA activity also ensured that the significantly higher RCA activity also ensured that Rubisco could be active in the plant. Under this treatment, the genes of carbon assimilation key enzymes rbcL and rbcS were significantly increased at the transcriptional level, increasing the amount of $\mathrm{CO}_{2}$ fixed by RuBP, thereby improving the carboxylation efficiency of Rubisco and the transportation efficiency of photosynthate. Therefore, enhanced UV-B radiation treatment can up-regulate the expression level of key enzyme genes of photosynthetic carbon assimilation in ' Guifei ' mango, promote the utilization rate of light energy, accelerate the Calvin cycle, and ultimately promote the photosynthetic efficiency, which may be an important mechanism for the growth and development of ' Guifei ' mango after $96 \mathrm{~kJ} \cdot \mathrm{m}^{-2} \cdot \mathrm{d}^{-1}$ enhanced UV-B radiation treatment.

\section{Conclusions}

Artificially simulated enhanced UV-B radiation treatment at $96 \mathrm{~kJ} \cdot \mathrm{m}^{-2} \cdot \mathrm{d}^{-1}$ can improve the photosynthetic pigments contents and chloroplast thylakoid stacking structure of 'Guifei' mango adult leaves, improve the ability of light energy capture, water photolysis, electron transfer and light energy conversion, enhance light reaction and form stronger assimilation. The treatment also increases the photosynthetic key enzymes activities by increasing the expressions of photosynthetic key enzymes genes such as $r b c L$ and $r b c S$, then enhances the $\mathrm{CO}_{2}$ fixation ability of leaves. Based on these two aspects, the enhanced UV-B radiation treatment increases the net photosynthetic rate of leaves, promotes the rapid accumulation of photosynthetic products, finally makes 'Guifei' mango ripe earlier and improved fruit quality. This paper mainly research advances about Photosynthetic Mechanism Regulated by $96 \mathrm{~kJ} \cdot \mathrm{m}^{-2} \cdot \mathrm{d}^{-1}$ enhanced UV-B radiation of 'Guifei' mango laid the early physiological foundation, and our findings is helpful to develop new technology of producing period regulation which is hard for 'Guifei' mango.

\section{References}

[1] Teramura,A.H. Effects of ultraviolet-B radiation on the growth and yield of crop plants. PhysiologiaPlantarum. 1983, 58(3):415-427.

[2] Rozema,J.; Van,D.; LO,Björn.; et al. UV-B as an environmental factor in plant life: stress and regulation. Trends in Ecology \& Evolution. 1997, 12(1):22-28.

[3] Krizek,D.T. Influence of PAR and UV-A in Determining Plant Sensitivity and Photomorphogenic Responses to UV-B Radiation ${ }^{\top \dagger}$, Photochemistry and Photobiology. 2004, 9(4): 307-315. 
[4] Neale,R.E.; Barnes,P.W.; Robson,T.M.; et al. Environmental effects of stratospheric ozone depletion, UV radiation, and interactions with climate change: UNEP Environmental Effects Assessment Panel, Update 2020. Photochemical \& Photobiological Sciences. 2021, 20(1):167.

[5] Crutzen.; Paul,J . Ultraviolet on the increase. Nature. 1992, 356(6365):104-105.

[6] Meng,F.; Bai,L.; Guo,H.; et al. Responses of different types of purple sweet potatoesin Yunnan low-latitude plateau to enhanced UV-B radiation. Journal of Tropical Agriculture. 2021, 41(02):24-32.

[7] Qi,W.; Ma,J.; Zhang,J.; Gui,M.; Li,J.; Zhang,L. Effects of low doses ofUV-B radiation supplementation on tuber quality in purple potato (Solanum tuberosum L.). Plant Signaling \& Behavior. 2020, 15(9):1783490

[8] Duarte-Sierra,A.; Hasan,S.; Angers,P.; et al. UV-B radiation hormesis in broccoli florets: Glucosinolatesand hydroxy-cinnamates are enhanced by UV-B in florets during storage. Postharvest Biology and Technology. 2020, 168:111278

[9] Chen,Z.; Ma,Y.; Weng,Y.; Yang,R.; Gu,Z.; Wang P. Effects of UV-B radiationon phenolic accumulation, antioxidant activity and physiological changes in wheat ( Triticum aestivum L.) seedlings. Food Bioscience. 2019, 30(26):100409

[10] Saber,H.; El-Sheekh,M.M.; Ibrahim,A.; et al. Effect of UV-B Radiation on Amino Acids Profile, Antioxidant Enzymes and Lipid Peroxidation of Some Cyanobacteria and Green Algae. International Journal of Radiation Biology. 2020, 96 (9):1-30.

[11] Ballaré,C.L.; Caldwell,M.M.; Flint,S.D.; Robinson,S.A.; Bornman,J.F. Effects of solar ultraviolet radiation onterrestrial ecosystems. Patterns, mechanisms, and interactions with climate change.Photochemical \& Photobiological Sciences Official Journal of the European Photochemistry Association \& the European Society for Photobiology. 2011, 10(2):226-241

[12] Albert,K.R.; Mikkelsen,T.N.; Ro-Poulsen,H. Ambient UV-B radiation decreases photosynthesis in higharctic Vaccinium uliginosum. Physiologia Plantarum. 2010, 133(2):199-210.

[13] V.G.KAKANI.; K.R.REDDY.D. ZHAO,A.R. MOHAMMED. Effects of Ultraviolet-B Radiation on Cotton ( Gossypium hirsutum L.) Morphology and Anatomy. Annals of Botany. 2003, 91(7):817-826.

[14] Qiang,J.; Chen,Y.; Effects of UV-B Radiation on Photosynthetic Characteristics and Pigment in Flue-Cured Tobacco Leaves. Hans Joural of Agricultural Sciences. 2019, 9(7):527-535

[15] Zhang,X.; Tang,X.; Zhou,B.; et al. Effect of enhanced UV-B radiation on photosyntheticcharacteristicsof marine microalgae Dunaliella salina (Chlorophyta, Chlorophyceae). Journal of Experimental Marine Biology\&Ecology. 2015, 469:27-35.

[16] Liu,Y.; Zhang,C.; Differential response of leaf gas exchange to enhanced ultraviolet-B (UV-B) radiation in three species of herbaceous climbingplants. Acta Ecologica Sinica. 2009, 29: 124-129.

[17] J,Martínez-Lüscher.; Morales,F.; Delrot,S.; et al. Short- and long-term physiological responses of grapevineleaves to UV-B radiation. Plant Science. 2013, 213(Complete): 114-122.

[18] Wang,H.; Yang,C.; Yue,K.; et al. The effects of different UV-B radiation on mango fruitquality and antioxidant properties. Chinese Journal of Tropical Crops. 2020, 41(02): 275-283.

[19] Wang,H.; Yue,K.; Yang,C.; et al. Physiological reasons for the inhibition of photosynthesis of mango leaves by enhanced UV-B radiation treatment. Acta Horticulture. 2020, 47(02): 242-252.

[20] Zhou,K.; Li,S.; Yuan,M.; Effects of enhanced UV-B radiation on mango plant yield, fruit quality and photosynthesis. Chinese Journal of Tropical Crops. 2019, 39(06):1102-1107.

[21] Li,H. Principles and techniques of plant physiological and biochemical experiments. 2000, Beijing:HigherEducation Press:134-138.

[22] Arnon,I. Copper enzymes in isolated chloroplasts. Polyphenoloxidase in Beta vulgaris. PlantPhysiology. 1949, 24(1):1-15.

[23] Yu,S.; Zheng,G.; A preliminary study on the Hill reaction activity of wheat chloroplasts. Journal of Shandong Agricultural College. 1980, $02: 45-52$

[24] Zhang,Z.; Zhu,Q.; Hu,M.; et al. Low-temperature conditioning induces chilling tolerancein stored mango fruit. Food Chemistry. 2017, 219:7684.

[25] Cutraro,J.; Cleaning Up Contaminants With Plants. business, 2005.

[26] Choi,B.Y.; Roh,K.S.; UV-B radiation affects chlorophyll and activation of rubisco by rubisco activase in Canavalia ensiformis L. leaves. Journal of Plant Biology. 2003, 46(2):117-121.

[27] Liu,Z.; Ma,D.; Zeng,S.; A study on the relation between chlorophyll content and photosynthetic rate ofrice. Acta Agronomica Sinica, 1984.

[28] Yang,Y.; Guo,S.; Yang,S.; etc. Effects of enhanced UV-B radiation on photosyntheticphysiology and ultramicrostructure of mango leaves. Acta Fructosa. 2021, 38(09):1524-1539.

[29] Liu,M.; Bo,C.; Zhou,S.; et al. Responses of the flavonoid pathway to UV-B radiation stress and the correlation with the lipid antioxidant characteristics in the desert plant Caryopteris mongolica. Acta Ecologica Sinica, 2012, 32(3):150-155.

[30] Chao,H.; Liu,Q.; Yang,Y.; Short-term effects of experimental warming and enhanced ultraviolet-B radiation on photosynthesis and antioxidant defense of Picea asperata seedlings. Plant Growth Regulation, 2009, 58(2):153-162.

[31] Surabhi,G.K.; Reddy,K.R.; Singh,S.K.; Photosynthesis, fluorescence, shoot biomass andseed weight responses of three cowpea (Vigna unguiculata (L.) Walp.) cultivars with contrasting sensitivity to UV-B radiation. Environmental \& Experimental Botany, 2009, 66(2):160-171.

[32] Teramura,A.H.; Ziska,L.H.; Sztein,A.E.; Changes in growth and photosynthetic capacityof rice with increased UV-B radiation. Physiologia Plantarum, 2010, 83(3):373 - 380.

[33] Basiouny,F.M.; Van,T.K.; Biggs,R.H.; Some Morphological and Biochemical Characteristics ofC3 and C4 Plants Irradiated with UV-B. Physiologia Plantarum, 2010, 42(1): 29-32.

[34] Li,T.; Yamane,H.; Tao,R.; Preharvest long-term exposure to UV-B radiation promotes fruit ripening and modifies stage-specific anthocyanin metabolism in highbush blueberry. Horticultureresearch, 2021, 8(1):67-67.

[35] Fang,F.; Wang,F.; Effect of UV-B irradiation on fruit quality and flavonol accumulation of postharvest grape berry. Science and Technology of Food Industry. 2017, 38(19):272-277+283.

[36] Mishra,V.; Srivastava,G.; Prasad,S.M.; et al. Growth, photosynthetic pigments and photosynthetic activity during seedling stage of cowpea (Vigna unguiculata) in response to UV-B and dimethoate. Pesticide Biochemistry \& Physiology, 2008, 92(1):30-37.

[37] Gonzalez-Mendoza,D.; Francisco,E.; Santamaría,J.M.; et al. Multiple Effects of Cadmium on the Photosynthetic Apparatus of Avicennia germinans L. as Probed by OJIP ChlorophyllFluorescence Measurements.Zeitschrift Für Naturforschung C, 2007, 62(3-4):265-272.

[38] Zavafer,A.; Koinuma,W.; Chow,W.S.; et al. Mechanism of Photodamage of the Oxygen Evolving Mn Cluster of Photosystem II by Excessive Light Energy. Scientific Reports. 2017, 7(1):7604.

[39] Liang,C.; Huang,X.;Zhou,Q.; Effect of cerium on photosynthetic characteristics of soybean seedling exposed to supplementary ultraviolet-B radiation. Journal of Environmental Sciences, 2006,18(6):1147-1151

[40] Miziorko,H.M.; Lorimer,G.H.; Ribulose-1,5-bisphosphate carboxylase-oxygenase. Annual Reviewof Biochemistry, 1983, $28(1): 507-535$.

[41] Cai,Z.; Liu,G.; Zhang,J.; Li,Y.; Development of an activity-directed selection system enabled significant improvement of the carboxylation efficiency of Rubisco. Protein \& cell. 2014, 5(7):552-562. 
[42] Vassileva,V.; Demirevska,K.; Simova-Stoilova,L.; et al. Long-Term Field Drought Affects Leaf Protein Pattern and Chloroplast Ultra structure of Winter Wheat in a Cultivar-Specific Manner. Journal of Agronomy and Crop Science. 2011, 198(2):104-117.

[43] Archie,R.; Portis.; et al. The regulation of Rubisco by Rubisco activase. Journal of Experimental Botany, 1995, 46(Special Issue):1285-1291. 